\title{
Clinical Characteristics and Risk Factors for Mortality in Older Patients with Dialysis-Receiving Community-Acquired Acute Kidney Injury
}

\author{
Guanglan $\mathrm{Li}^{1,2}$ \\ Xingji Lian' \\ Wenke Hao (D) \\ Wenxue $\mathrm{Hu}^{\prime}$ \\ 'Department of Nephrology, Guangdong \\ Provincial People's Hospital, Guangdong \\ Academy of Medical Sciences, Guangdong \\ Provincial Geriatrics Institute, Guangzhou, \\ 510080, People's Republic of China; \\ ${ }^{2}$ Shantou University Medical College, \\ Shantou, 5 I504I, People's Republic of \\ China
}

Introduction: Older people in community are susceptible to acute kidney injury (AKI) and hemodialysis is the most important supportive measure used in the management of severe AKI. This study aims to investigate the clinical characteristics, outcomes and risk factors for mortality in older patients with dialysis-receiving-community-acquired AKI (CA-AKI).

Methods: A total of 1953 CA-AKI patients aged 65 years old and above were recruited from 2013 to 2016. Among which, 200 patients received hemodialysis. Clinical characteristics, outcomes, suspected nephrotoxic drug use after CA-AKI and risk factors for mortality in older CA-AKI patients with dialysis were analyzed.

Results: The percentage of CA-AKI patients receiving hemodialysis was $10.2 \%$. Compared with non-dialysis patients, dialysis-receiving patients had more comorbidity, and worse renal function. The types of suspected nephrotoxic drugs used in dialysis patients were more than those in non-dialysis patients. Moreover, dialysis-receiving patients had worse outcomes, including complete recovery of renal function $(42.0 \%$ vs $71.6 \%)$, intensive care unit (ICU) $(69.0 \%$ vs $15.3 \%)$ transfer and in-hospital mortality $(50.5 \%$ vs $5.6 \%)(P<0.01)$. Age, moderate/ severe liver disease, beta lactam antibiotics, glycopeptide antibiotics, antifungal agents, drugs for anti-heart failure, category of suspected nephrotoxic drugs, hyperkalemia, increased leucocyte count, ICU transfer, multiple organ dysfunction (MODS), cardiogenic shock and cardio-pulmonary resuscitation (CPR) were risk factors for mortality by univariate logistic regression analysis. After adjusting for confounding factors, the independent risk factors were glycopeptide antibiotics, drugs for anti-heart failure, ICU transfer, MODS and CPR.

Conclusion: The percentage of older CA-AKI patients receiving dialysis was high, and these patients had more comorbidity and worse prognosis. Glycopeptide antibiotics, drugs for anti-heart failure, ICU transfer, MODS and CPR were independent risk factors for mortality.

Keywords: community-acquired acute kidney injury, older people, dialysis, outcome, risk factors for mortality

\section{Introduction}

Acute kidney injury (AKI) is common in hospitalized patients, ${ }^{1}$ which can be divided into community-acquired AKI (CA-AKI) when a patient had AKI in community and hospital-acquired AKI (HA-AKI) occurring during hospitalization. ${ }^{2}$ A study showed that the incidence of CA-AKI and HA-AKI was $4.3 \%$ and $2.1 \%$, respectively. ${ }^{3}$ Our recent study showed the incidence of CA-AKI was $6.51 \%$ in patients who were 60 years old and above, ${ }^{4}$ and another research revealed the incidence was $10.47 \%$ in 
patients aged 65 years old and above. ${ }^{5}$ Baraldi et al also found that the incidence in AKI was 10 times higher in those aged over 65 as compared with those under 65 years old. ${ }^{6}$ Therefore, CA-AKI in older patients is prevalent and understanding the characteristics of CA-AKI is very important for clinicians.

Renal replacement therapy (RRT) is one of the most important and effective treatment plans in severe AKI patients. ${ }^{7}$ Recent studies showed that the incidence of dialysis-receiving AKI increased rapidly in adults in the past decade. ${ }^{8,9}$ Older patients had higher incidence of dialysis-receiving AKI, and then patients suffering from AKI requiring dialysis had higher mortality. ${ }^{9}$ By far, few studies focus on the clinical features of CA-AKI patients with dialysis. Therefore, we conducted a retrospective study of older patients with dialysis-receiving CA-AKI, investigating the clinical characteristics, outcomes and risk factors for in-hospital mortality.

\section{Materials and Methods Study Design and Data Source}

This was a population-based retrospective cohort study of older patients who were treated with hemodialysis for CAAKI in Guangdong Provincial People's Hospital from 2013 to 2016. We conducted this study through using electronic medical record databases in Guangdong Provincial People's Hospital.

\section{Study Population}

The study included older inpatients aged $\geq 65$ years old with dialysis-receiving CA-AKI in Guangdong Provincial People's Hospital. Exclusion criteria: (1) Patients with preexisting chronic kidney disease (CKD) stage 5; (2) Patients receiving any dialysis or kidney transplantation before the study entry date; (3) Patients with hospitalization for more than 30 days; (4) Patients with missing data. The study involving human participants was approved by the Ethical Committee of Guangdong Provincial People's Hospital (GDREC2016327H; Guangdong, China).

\section{Definition of Diseases}

AKI was defined by 2012 KDIGO AKI guidelines: an increase in serum creatinine ( $\mathrm{SCr}$ ) by $0.3 \mathrm{mg} / \mathrm{dL}$ within 48 hours or a $50 \%$ increase in $\mathrm{SCr}$ within 7 days. ${ }^{10} \mathrm{CA}$ AKI was defined by SCr change on the first day of admission met the KDIGO definition.
Baseline serum creatinine values for patients with CAAKI were determined by reviewing all Scr values of the patient (from the hospital or the community) over the previous 12 months. In addition, patients with unknown baseline values were plotted with Scr values after AKI recovery to further approximate the baseline Scr and confirm whether it was true AKI.

The Scr value at discharge was less than 1.2-fold baseline for full renal recovery, the Scr value at discharge was 1.2-1.5-fold baseline for partial renal recovery, and the Scr value at discharge was greater than 1.5 -fold baseline or renal replacement therapy for nonrecovery.

\section{Determination of Comorbidity}

Comorbidity was determined by the diagnosis codes according to International Classification of Disease (ICD): (1) Myocardial infarction: I21; I22; I25.2. (2) Congestive heart failure: I50; I11.0; I13.0; I13.2. (3) Peripheral vascular disease: I73; I73.8; I73.9; I77.9 et al (4) Cerebrovascular disease: I60; I61; I62; I63; I65 et al (5) Dementia: F00; F01; F02; FO3 et al (6) Chronic pulmonary disease: J41; J42; J44.8 et al (7) Connective tissue disease: M32; M34; M33.2; M33.1; M05.3 et al (8) Ulcer disease: K25; K26; K27; K28; K22.1. (9) Mild liver disease: B18; K70; K73 et al (10) Moderate/severe liver disease: K72.9; K72.1; K70.4 et al (11) Diabetes without complications: E10.9; E11.9; E13.9; E14.9 et al (12) Diabetes with complications: E10.2; E11.2; E13.2; E14.2; E10.3 et al (13) Malignant tumor: C77-C80. The burden of comorbidity was determined using Charlson co-morbidity index (CCI). ${ }^{11}$

\section{Statistical Analyses}

Statistical analyses were carried out using SPSS (version 25.0; SPSS, Inc., Chicago, IL, USA). Mann-Whitney $U$-test was used for analysis of not normally distributed data. Pearson chi-squared test was used to compare categorical data. Logistic regression analysis was used to identify independent predictors of in-hospital mortality for older dialysis-receiving CA-AKI patients. All the variables with statistical significance by univariable analysis were included in a multivariable analysis model, including age, moderate or severe liver disease, diabetes with complications, beta lactam antibiotics, antifungal agents, diuretic and dehydrating agents, angiotensin converting enzyme inhibitors (ACEIs) or angiotensin II receptor blockers (ARBs), non-steroidal anti-inflammatory drugs (NSAIDS), drugs for anti-heart failure, category of suspected nephrotoxic drugs, hyperkalemia, 
hypoalbuminema, increased leucocyte count, intensive care unit (ICU) transfer, multiple organ dysfunction (MODS), cardiogenic shock, and cardio-pulmonary resuscitation (CPR). $P$ value $<0.05$ was considered to represent statistically significant differences.

\section{Results}

\section{The Clinical Characteristics of Dialysis-Receiving CA-AKI in Older Patients}

In this study, a total of 1953 CA-AKI patients aged $\geq 65$ years old were identified, of which 200 patients were treated with hemodialysis. The dialysis rate of CA-AKI in older patients was $10.2 \%$.

The majority of patients were male $(69.5 \%)$, and the mean age of CA-AKI patients with dialysis was 77 years old, ranging from 71 to 81 years old, there was no difference in age between dialysis and non-dialysis patients $(P>0.05)$. Compared with CA-AKI patients without dialysis, dialysis-receiving patients had more comorbidity (CCI: 4.0 vs 3.0 score, $P=0.000$ ), the percentages of preexisting $\mathrm{CKD}$, myocardial infarction, congestive heart failure, moderate/severe liver disease and diabetes were $21.0 \%$ (42 cases), $22.0 \%$ (44 cases), $52.5 \%$ (105 cases), $7.0 \%$ (14 cases), $39.0 \%$ (78 cases), respectively, of patients with dialysis-receiving CA-AKI, which were higher than that of patients without dialysis-receiving CAAKI $(P<0.05)$. Baseline characteristics, including age, sex, CCI, history of diseases are shown in Table 1.

According to laboratory data, we found that dialysisreceiving patients had higher $\mathrm{SCr}$ levels, both the maximum and baseline $\mathrm{SCr}(352.50$ vs $129.00 \mu \mathrm{mol} / 1,123.75$ vs $67.90 \mu \mathrm{mol} / 1$, respectively, $P=0.000$ ). Furthermore, the percentages of increased leucocyte count, low carbon dioxide binding force and hyperkalemia in dialysis patients were higher than that of non-dialysis patients (77.5\% vs $44.4,93.5 \%$ vs $57.8,62.0 \%$ vs $16.5 \%$, respectively, $P=0.000$ ). Laboratory data are shown in Table 2 .

\section{The Use of Suspected Nephrotoxic Drugs in Post-CA-AKI Patients}

After classifying the suspected nephrotoxic drugs, we found that the proportion of patients received 1 to 3 drugs in non-dialysis group was higher than that in

Table I The Baseline Characteristics of CA-AKI Patients with Dialysis or without Dialysis in Older Patients

\begin{tabular}{|c|c|c|c|}
\hline Variable & Non-Dialysis Group & Dialysis Group & $P$ value \\
\hline Cases & 1753 & 200 & \\
\hline Age (year), M (I/4, 3/4) & $77(7 \mathrm{I}, 83)$ & $77(7 \mathrm{I}, 8 \mathrm{I})$ & 0.611 \\
\hline Men, n (\%) & $1105(63.0)$ & $139(69.5)$ & 0.072 \\
\hline \multicolumn{4}{|l|}{ Past history n (\%) } \\
\hline Chronic kidney disease & $129(7.4)$ & $42(21.0)$ & $0.000^{*}$ \\
\hline Hypertension & $1032(58.9)$ & $127(63.5)$ & 0.207 \\
\hline Myocardial infarction & $177(10.1)$ & $44(22.0)$ & $0.000^{*}$ \\
\hline Congestive Heart failure & $510(29.1)$ & $105(52.5)$ & $0.000 *$ \\
\hline Peripheral vascular disease & $530(30.2)$ & $5 \mathrm{I}(25.5)$ & 0.165 \\
\hline Cerebrovascular disease & $530(30.2)$ & $5 \mathrm{I}(25.5)$ & 0.165 \\
\hline Dementia & $80(4.6)$ & $4(2.0)$ & 0.090 \\
\hline Chronic pulmonary disease & $460(26.2)$ & $37(18.5)$ & $0.017^{*}$ \\
\hline Connective tissue disease & $113(6.4)$ & $14(7.0)$ & 0.763 \\
\hline Ulcer disease & $95(5.4)$ & $7(3.5)$ & 0.248 \\
\hline Mild liver disease & $916(52.3)$ & $115(57.5)$ & 0.159 \\
\hline Moderate/severe liver disease & $45(2.6)$ & $14(7.0)$ & $0.001 *$ \\
\hline Diabetes without complications & $542(30.9)$ & $78(39.0)$ & $0.020^{*}$ \\
\hline Diabetes with complications & $73(4.2)$ & $26(13)$ & $0.000^{*}$ \\
\hline Malignant tumor & $217(12.4)$ & $20(10.0)$ & 0.329 \\
\hline Charlson comorbidity index score $(\mathrm{CCl}), \mathrm{M}(\mathrm{I} / 4,3 / 4)$ & $3.0(2.0,5.0)$ & $4.0(2.0,6.75)$ & $0.000^{*}$ \\
\hline $\mathrm{CCl} \geq 3$ score & $1003(57.2)$ & $149(74.5)$ & $0.000 *$ \\
\hline
\end{tabular}

Note: $* P<0.05$.

Abbreviation: CA-AKI, Community-acquired acute kidney injury; Non-dialysis, CA-AKI patients without dialysis. 
Table 2 The Clinical Characteristics of CA-AKI Patients with Dialysis or Without Dialysis in Older Patients

\begin{tabular}{|l|l|l|l|}
\hline \multirow{2}{*}{ Variables } & Non-Dialysis Group & Dialysis Group & \multirow{2}{*}{ P value } \\
\cline { 2 - 3 } & $\mathbf{( n = I 7 5 3 )}$ & $\mathbf{( n = 2 0 0 )}$ & \\
\hline Maximum SCr (umol/I), M (I/4,3/4) & $129.00(92.90,189.50)$ & $352.50(266.37,501.27)$ & $0.000^{*}$ \\
Baseline SCr (umol/I), M (I/4, 3/4) & $67.90(50.00,93.65)$ & $123.75(86.32,181.00)$ & $0.000^{*}$ \\
Hypoalbuminema n (\%) & $486(27.7)$ & $54(27.0)$ & 0.828 \\
Increased leucocyte count n (\%) & $778(44.4)$ & $155(77.5)$ & $0.000^{*}$ \\
Low carbon dioxide binding force $\mathrm{n}(\%)$ & $1013(57.8)$ & $187(93.5)$ & $0.000^{*}$ \\
Hyperkalemia n (\%) & $290(16.5)$ & $124(62.0)$ & $0.000^{*}$ \\
\hline
\end{tabular}

Note: $* p<0.05$.

Abbreviation: CA-AKI, Community-acquired acute kidney injury; Non-dialysis, CA-AKI patients without dialysis.

dialysis group ( $84.0 \%$ vs $60.5 \%, P=0.000$ ), whereas the proportion of patients received more than three medications in dialysis group was higher than that in nondialysis group $(39.5 \%$ vs $15.9 \%, P=0.000)$. The percentage of antibiotic use was higher in dialysis-receiving patients than that in non-dialysis patients, including beta lactam antibiotics $(75.5 \%$ vs $62.9 \%, P=0.000)$, glycopeptide antibiotics $(12.5 \%$ vs $3.4 \%, P=0.000)$, antifungal agents $(4.5 \%$ vs $1.0 \%, P=0.000)$, but there was no difference of aminoglycosides between two groups
$(P>0.05)$. The proportions of application for ACEIs or ARBs were significantly decreased in patients with dialysis when compared with non-dialysis $(P=0.000)$. However, the proportions of other possible nephrotoxic drugs in the present study for concern (including adrenergic receptor agonists and drugs for anti-heart failure) were still increased in dialysis-receiving CA-AKI patients $(P=0.000)$. The use of suspected nephrotoxic drugs in post-CA-AKI older patients with dialysis is listed in Table 3.

Table 3 Suspected Nephrotoxic Drugs Use in Post-CA-AKI Older Patients with Dialysis or without Dialysis

\begin{tabular}{|l|c|c|c|}
\hline \multirow{2}{*}{ Suspected Nephrotoxic Drugs [Case (\%)] } & Non-Dialysis Group & Dialysis Group & \multirow{2}{*}{ P value } \\
\cline { 2 - 4 } & $\mathbf{( n = 1 7 5 3 )}$ & $15(7.5)$ & 0.107 \\
\hline Aminoglycosides & $85(4.8)$ & $151(75.5)$ & $0.000 *$ \\
\hline Beta lactam antibiotics & $1103(62.9)$ & $25(12.5)$ & $0.000 *$ \\
\hline Glycopeptide antibiotics & $59(3.4)$ & $9(4.5)$ & $0.000 *$ \\
\hline Antifungal agents & $17(1.0)$ & $97(48.5)$ & 0.098 \\
\hline Diuretic and dehydrating agents & $743(42.4)$ & $55(27.5)$ & $0.000 *$ \\
\hline ACEls or ARBs & $773(44.1)$ & $1(0.5)$ & 0.136 \\
\hline Chemotherapeutic agents & $35(2.0)$ & $100(50.0)$ & 0.630 \\
\hline $\begin{array}{l}\text { NSAIDS } \\
\text { Ferralia }\end{array}$ & $908(51.8)$ & $3(1.5)$ & 0.597 \\
\hline Adrenergic receptor agonists & $19(1.1)$ & $150(75.0)$ & $0.000 *$ \\
\hline Drugs for anti-heart failure & $350(20.0)$ & $16(8.0)$ & $0.000 *$ \\
\hline $\begin{array}{l}\text { Category of suspected nephrotoxic drugs } \\
\text { I-3 categories } \\
>3 \text { categories }\end{array}$ & $27(1.5)$ & $\begin{array}{l}121(60.5) \\
79(39.5)\end{array}$ \\
\hline
\end{tabular}

Note: $* P<0.05$.

Abbreviations: CA-AKI, Community-acquired acute kidney injury; Non-dialysis, CA-AKI patients without dialysis; ACEls or ARBs, Angiotensin converting enzyme inhibitors or angiotensin receptor antagonists; NSAIDS, Non-steroidal anti-inflammatory drugs. 


\section{Clinical Outcomes in Older CA-AKI Patients with Dialysis}

The prognosis of dialysis-receiving CA-AKI patients was poor. The inpatient all-cause mortality of patients with $\mathrm{CA}$ AKI was $10.2 \%$. In-hospital mortality was higher in dialysis patients than that of non-dialysis patients $(50.5 \%$ vs $5.6 \%$, $P=0.000)$. The percentages of MODS (35.5\% vs $2.7 \%$, $P=0.000)$ and cardiogenic shock ( $10.0 \%$ vs $0.6 \%, P=0.000)$ in dialysis patients were higher during hospitalization. In addition, compared with non-dialysis patients, the proportions of patients with dialysis who needed to transfer to ICU (69.0\% vs $15.3 \%)$, CPR (41.5\% vs $2.5 \%)$ and intra-aortic balloon pump (IABP) (8.0\% vs 1.4\%) during hospitalization were higher $(P=0.000)$. Furthermore, the proportion of fully renal recovery in non-dialysis patients was higher than that of dialysis patients $(71.6 \%$ vs $42.0 \%, P=0.000)$, while the proportions of partial renal recovery $(19.0 \%$ vs $17.5 \%, P=0.000)$ and non-recovery $(39.0 \%$ vs $10.9 \%, P=0.000)$ in dialysis patients were higher than that of non-dialysis patients. Clinical outcomes in CA-AKI patients with dialysis and nondialysis are shown in Table 4.

\section{Risk Factors for Mortality in Older CA-AKI Dialysis Patients}

For high mortality of older CA-AKI dialysis patients $(50.5 \%)$, the risk factors for mortality in older CA-AKI dialysis patients were analyzed. Single factor logistic regression analysis indicated that the risk factors for mortality were as follows: age, moderate/severe liver disease, beta lactam antibiotics, glycopeptide antibiotics, antifungal agents, drugs for anti-heart failure, category of suspected nephrotoxic drugs, hyperkalemia, increased leucocyte count, ICU transfer, MODS, cardiogenic shock and CPR. The detailed results of the univariate exact logistic regression analyses are shown in Table 5.

Accounting for the effects of other variables, we further used multivariable analysis model. The results showed that glycopeptide antibiotics (OR 6.339, 95\% CI $1.009,39.835)$, drugs for anti-heart failure (OR 23.982, 95\% CI 2.687, 214.047), ICU transfer (OR 10.813, 95\% CI 2.917, 40.091), MODS (OR 5.719, 95\% CI 2.078, 15.740), and CPR (OR 38.796, 95\% CI 12.885, 116.812) were independent risk factors for mortality in older CAAKI dialysis patients. The detailed results of the multivariable logistic regression analyses are shown in Table 5.

\section{Discussion}

Dialysis is an important treatment for AKI. Xu et al indicated that $4.5 \%$ of patients in adult with CA-AKI required dialysis during hospitalization. ${ }^{12}$ Wonnacott et al showed that $3.5 \%$ of patients in adult with $\mathrm{CA}-\mathrm{AKI}$ required inpatient RRT. ${ }^{3}$ In this study, we found the rate of dialysis in older patients with CA-AKI was $10.2 \%$. These data indicated that the rate of dialysis of CA-AKI in older patients was higher than that in younger adults. This phenomenon may be explained by the following data. Older patients had more comorbidity, including CKD. The prevalence of CKD is almost three to four times higher in older adults than younger adults. ${ }^{13}$ When the elderly develop CKD they have less kidney functional reserve. ${ }^{14}$ Moreover, more comorbidity means higher chance using nephrotoxic drugs which would aggravate renal damage.

Table 4 Clinical Outcomes in CA-AKI Patients with Dialysis or without Dialysis in Older Patients

\begin{tabular}{|l|l|c|c|}
\hline \multirow{2}{*}{ Clinical Outcomes } & Non-Dialysis Group & Dialysis Group & P value \\
\cline { 2 - 4 } & (n=1753) & (n=200) & $0.000^{*}$ \\
\hline In-hospital mortality (n, \%) & $99(5.6)$ & $101(50.5)$ & $0.000^{*}$ \\
MODS (n, \%) & $47(2.7)$ & $71(35.5)$ & $0.000^{*}$ \\
Cardiogenic shock (n, \%) & II (0.6) & $20(10.0)$ & $0.000^{*}$ \\
ICU transfer (n, \%) & $268(15.3)$ & $138(69.0)$ & $0.000^{*}$ \\
Renal recovery (n, \%) & & & \\
Complete recovery & $1256(71.6)$ & $84(42.0)$ & \\
Partial recovery & $306(17.5)$ & $38(19.0)$ & $0.000^{*}$ \\
No recovery & $191(10.9)$ & $78(39.0)$ & $0.000^{*}$ \\
CPR (n, \%) & $43(2.5)$ & $16(8.0)$ & \\
IABP (n, \%) & $25(1.4)$ & & \\
\hline
\end{tabular}

Note: $* P<0.05$.

Abbreviations: CA-AKI, Community-acquired acute kidney injury; Non-dialysis, CA-AKI patients without dialysis; MODS, Multiple organ dysfunction; ICU, Intensive care unit; CPR, Cardio-Pulmonary resuscitation; IABP, Intra-aortic balloon pump. 
Table 5 Logistic Regression Analyses of Risk Factors for Mortality in Older CA-AKI Dialysis Patients

\begin{tabular}{|c|c|c|c|c|}
\hline \multirow[t]{2}{*}{ Variables } & \multicolumn{2}{|l|}{ Univariate Analysis } & \multicolumn{2}{|l|}{ Multivariate Analysis } \\
\hline & OR (95\% Cl) & $P$ value & OR (95\% Cl) & $P$ value \\
\hline Sex & $1.353(0.793-2.478)$ & 0.327 & & \\
\hline Age & 1.063 (I.019-I.109) & $0.004 *$ & & \\
\hline Chronic kidney disease & $0.033(0.230-0.94 I)$ & 0.465 & & \\
\hline Hypertension & $0.586(0.328-1.050)$ & 0.073 & & \\
\hline Myocardial infarction & $0.975(0.499-1.903)$ & 0.940 & & \\
\hline Congestive Heart failure & $0.724(0.4|5-| .263)$ & 0.255 & & \\
\hline Peripheral vascular disease & $1.410(0.743-2.675)$ & 0.293 & & \\
\hline Cerebrovascular disease & $1.410(0.743-2.675)$ & 0.293 & & \\
\hline Dementia & $3.000(0.307-29.343)$ & 0.345 & & \\
\hline Chronic pulmonary disease & $1.362(0.663-2.795)$ & 0.400 & & \\
\hline Connective tissue disease & $0.718(0.240-2.151)$ & 0.555 & & \\
\hline Ulcer disease & $1.320(0.288-6.053)$ & 0.721 & & \\
\hline Mild liver disease & $0.606(0.344-1.068)$ & 0.083 & & \\
\hline Moderate/severe liver disease & I4.477 (I.856-I I2.933) & $0.011^{*}$ & & \\
\hline Diabetes without complications & $0.582(0.328-1.034)$ & 0.065 & & \\
\hline Diabetes with complications & $0.193(0.070-0.537)$ & $0.002 *$ & & \\
\hline Malignant tumor & $2.494(0.918-6.780)$ & 0.073 & & \\
\hline Aminoglycosides & $2.903(0.892-9.448)$ & 0.077 & & \\
\hline Beta lactam antibiotics & $3.398(1.690-6.853)$ & $0.00 I^{*}$ & & \\
\hline Glycopeptide antibiotics & 3.591 (1.369-9.424) & $0.009 *$ & $6.339(1.009-39.835)$ & 0.049 \\
\hline Antifungal agents & $8.430(1.034-68.713)$ & $0.046^{*}$ & & \\
\hline Diuretic and dehydrating agents & $0.345(0.194-0.613)$ & $0.000 *$ & & \\
\hline ACEls or ARB & $0.363(0.189-0.698)$ & $0.002^{*}$ & & \\
\hline NSAIDS & $0.697(0.399-1.216)$ & $0.032 *$ & & \\
\hline Drugs for anti-heart failure & $7.805(1.725-35.315)$ & $0.000 *$ & $23.982(2.687-2 \mid 4.047)$ & 0.004 \\
\hline Category of suspected & $1.577(1.182-2.104)$ & $0.002^{*}$ & & \\
\hline \multicolumn{5}{|l|}{ Nephrotoxic drugs } \\
\hline Hyperkalemia & $1.882(1.055-3.358)$ & $0.032^{*}$ & & \\
\hline Hypoalbuminema & $0.424(0.222-0.809)$ & $0.009 *$ & & \\
\hline Increased leucocyte count & $4.280(2.020-9.069)$ & $0.000 *$ & & \\
\hline Low carbon dioxide binding & $1.688(0.533-5.350)$ & 0.374 & & \\
\hline ICU transfer & $13.950(6 .|23-31.78|)$ & $0.000 *$ & $10.813(2.917-40.091)$ & 0.000 \\
\hline
\end{tabular}

(Continued) 
Table 5 (Continued).

\begin{tabular}{|l|l|l|l|l|}
\hline Variables & \multicolumn{2}{l|}{ Univariate Analysis } & \multicolumn{2}{l|}{ Multivariate Analysis } \\
\hline & OR $(95 \% \mathrm{CI})$ & P value & OR (95\% CI) & $P$ value \\
\hline MODS & $13.950(6.123-31.78 I)$ & $0.000^{*}$ & $5.719(2.078-15.740)$ & 0.001 \\
\hline Cardiogenic shock & $6.202(3.196-12.038)$ & $0.004^{*}$ & & \\
\hline CPR & $39.954(16.383-97.438)$ & $0.000^{*}$ & $38.796(12.885-116.812)$ & 0.000 \\
\hline
\end{tabular}

Note: $* P<0.05$.

Abbreviations: CA-AKI, Community-acquired acute kidney injury; ACEls or ARBs, Angiotensin converting enzyme inhibitors or angiotensin receptor antagonists; NSAIDS, Non-steroidal anti-inflammatory drugs; ICU, Intensive care unit; MODS, Multiple organ dysfunction; CPR, Cardio-Pulmonary resuscitation.

The optimal timing of renal replacement therapy is important for AKI. Our study found CA-AKI patients had higher SCr levels, both the maximum and baseline $\mathrm{SCr}$ in dialysis patients than that in non-dialysis patients. In addition, increased leucocyte count, low carbon dioxide binding force and hyperkalemia were more prevalent in dialysis-receiving patients. Similarly, Wilson et al matched dialysis with non-dialysis patients and analyzed the clinical characteristics of two groups. The results showed the initiation of dialysis was strongly related to higher levels of SCr, potassium, and blood urea nitrogen. ${ }^{15}$ These data indicated that dialysis-receiving patients had worse renal function, electrolyte disturbances and acid-base imbalances, which were indications for urgent dialysis.

Although dialysis is an important treatment for AKI, the prognosis of dialysis-receiving patients with CA-AKI in older patients is not well known. A study of children showed that patients with dialysis-receiving AKI had a higher rate of mortality than those with AKI not receiving dialysis $(27.1 \%$ vs $14.2 \%) .{ }^{16}$ In the same way, in the older patients with CA-AKI, we found a higher mortality rate in dialysis patients than in non-dialysis patients $(50.5 \%$ vs $5.6 \%$ ). In face of such a high mortality rate, we further investigated the risk factors for mortality. The results showed that age, moderate/severe liver disease, beta lactam antibiotics, glycopeptide antibiotics, antifungal agents, drugs for anti-heart failure, category of suspected nephrotoxic drugs, hyperkalemia, increased leucocyte count, ICU transfer, MODS, cardiogenic shock and CPR were risk factors for mortality by single factor logistic regression analysis. After adjusting for confounding factors, the independent risk factors were glycopeptide antibiotics, drugs for anti-heart failure, ICU transfer, MODS, and CPR.

It could be seen that increased leucocyte count and antibiotics (including beta lactam antibiotics, glycopeptide antibiotics, antifungal agents) were risk factors for mortality in older CA-AKI dialysis patients. As we know, infection was one of important etiology of CA-AKI, ${ }^{17}$ and the CA-AKI patients with infection had worse outcome than that of patients without infection. ${ }^{18}$ Our data showed that dialysis patients had higher leucocyte count and antibiotic use. And those indicated that more dialysis patients affected with infection, which was one of the reasons for mortality. Interestingly, among antimicrobial drugs, there was no statistical significance between aminoglycosides and mortality. Beta lactam antibiotics and glycopeptide antibiotics were risk factors for mortality, but only glycopeptide antibiotics was the independent risk factor for mortality. As known to physicians, nephrotoxicity was the main adverse effect of aminoglycosides. ${ }^{19}$ Therefore, aminoglycosides might be avoided in those AKI patients. ${ }^{20}$ In fact, aminoglycosides were used only in a small proportion of dialysis patients in this study, which may be the reason there was no statistical significance between aminoglycosides and mortality. Furthermore, vancomycin use was associated with higher incidence of AKI when $\mathrm{SCr}>2 \mathrm{mg} / \mathrm{dl},{ }^{21}$ and vancomycin was an independent risk factor for mortality in older CAAKI dialysis patients. Therefore, clinician should pay high attention to the choice of antibiotic, reduce doses in older patients with dialysis-receiving CA-AKI.

Potassium disorders were associated with adverse outcomes in various medical conditions. Arora et al demonstrated that preoperative hyperkalemia was associated with 30-day mortality, ${ }^{22}$ Kovesdy et al demonstrated that serum potassium $>5.5 \mathrm{mmol} / 1$ was associated with increased mortality in maintenance hemodialysis patients. ${ }^{23}$ Our study demonstrated that hyperkalemia was not only associated with the initiation of dialysis (as mentioned above), but was also a risk factor for mortality after dialysis. These data suggested that serum potassium level may be an effective predictor of dialysis-receiving CA-AKI prognosis. 
In addition, we also found that moderate/severe liver disease, drugs for anti-heart failure, ICU transfer, MODS, and CPR were risk factors for mortality in older patients with dialysis-receiving CA-AKI. Part results are similar to another study, which demonstrated that liver disease and ICU transfer were strong predictors of death in patients with $\mathrm{AKI} .{ }^{24} \mathrm{We}$ suspect that liver disease may aggravate metabolic disturbance to worse prognosis in these older CA-AKI patients. Moreover, our data above indicated that patients treated with anti-heart failure drug had worse cardiac function, the latter would increase the risk of death. Lastly, CA-AKI patients with ICU transfer, MODS, and CPR were critical ill patients, who were known for high mortality.

In this study, we conducted a research on CA-AKI with dialysis or non-dialysis in older patients and some significant findings were found. However, our study also has some limitations. Firstly, the subjects of this study were inpatients, so our data only reflected those hospitalized patients with more severe CA-AKI. Secondly, this study is a retrospective study and the follow-up time is short, so further prospective studies are needed to establish more reliable conclusions.

\section{Conclusions}

In conclusion, the percentage of dialysis in older CA-AKI patients was high, and these dialysis patients had more comorbidity, more kinds of suspected nephrotic drug use and worse prognosis. Glycopeptide antibiotics, drugs for anti-heart failure, ICU transfer, MODS and CPR were independent risk factors for mortality. These results may help to identify early risk factors for poor prognosis in dialysis-receiving CA-AKI patients and make therapeutic interventions to improve the prognosis.

\section{Ethical Approval}

The study involving human participants was approved by the Ethical Committee of Guangdong Provincial People's Hospital, written informed consent was obtained from the patients before the enrollment. This study was conducted in accordance with the Declaration of Helsinki.

\section{Acknowledgments}

We thank all patients and support staff in the study. Wenke Hao and Wenxue Hu are co-correspondence authors for this study.

\section{Funding}

This work was supported by the Natural Science Foundation of Guangdong Province, China [grant numbers 2019A1515011594, 2018A0303130251].

\section{Disclosure}

The authors declare that they have no known competing financial interests or personal relationships that could have appeared to influence the work reported in this paper.

\section{References}

1. James MT, Hemmelgarn BR, Wiebe N, et al. Glomerular filtration rate, proteinuria, and the incidence and consequences of acute kidney injury: a cohort study. Lancet. 2010;376(9758):2096-2103. doi:10.1016/S0140-6736(10)61271-8

2. Robert L, Ficheur G, Gautier S, et al. Community-acquired acute kidney injury induced by drugs in older patients: a multifactorial event. Clin Interv Aging. 2019;14:2105-2113. doi:10.2147/CIA.S217567

3. Wonnacott A, Meran S, Amphlett B, Talabani B, Phillips A. Epidemiology and outcomes in community-acquired versus hospital-acquired AKI. Clin $J$ Am Soc Nephrol. 2014;9 (6):1007-1014. doi:10.2215/CJN.07920713

4. Hu W, Lian X, Lin J, et al. The incidence, characteristics, and use of suspected nephrotoxic drugs in elderly patients with community-acquired acute kidney injury. Clin Interv Aging. 2021;16:35-42. doi:10.2147/CIA.S286660

5. Ge S, Nie S, Liu Z, et al. Epidemiology and outcomes of acute kidney injury in elderly Chinese patients: a subgroup analysis from the EACH study. BMC Nephrol. 2016;17(1):136. doi:10.1186/s12882-016-0351-2

6. Baraldi A, Ballestri M, Rapanà $\mathrm{R}$, et al. Acute renal failure of medical type in an elderly population. Nephrol Dial Transplant. 1998;13(Suppl 7):25-29. doi:10.1093/ndt/13.suppl_7.25

7. Baldwin I, Naka T, Koch B, Fealy N, Bellomo R. A pilot randomised controlled comparison of continuous veno-venous haemofiltration and extended daily dialysis with filtration: effect on small solutes and acid-base balance. Intensive Care Med. 2007;33(5):830-835. doi:10.1007/s00134-007-0596-0

8. Wald R, McArthur E, Adhikari NK, et al. Changing incidence and outcomes following dialysis-requiring acute kidney injury among critically ill adults: a population-based cohort study. Am J Kidney Dis. 2015;65(6):870-877. doi:10.1053/j.ajkd.2014.10.017

9. Hsu RK, McCulloch CE, Dudley RA, Lo LJ, Hsu CY. Temporal changes in incidence of dialysis-requiring AKI. J Am Soc Nephrol. 2013;24(1):37-42. doi:10.1681/ASN.2012080800

10. Khwaja A. KDIGO clinical practice guidelines for acute kidney injury. Nephron Clin Pract. 2012;120(4):c179-184.

11. Charlson ME, Pompei P, Ales KL, MacKenzie CR. A new method of classifying prognostic comorbidity in longitudinal studies: development and validation. $J$ Chronic Dis. 1987;40(5):373-383. doi:10.1016/0021-9681(87)90171-8

12. Xu X, Nie S, Liu Z, et al. Epidemiology and clinical correlates of AKI in Chinese hospitalized adults. Clin J Am Soc Nephrol. 2015;10 (9):1510-1518. doi:10.2215/CJN.02140215

13. Zhang QL, Rothenbacher D. Prevalence of chronic kidney disease in population-based studies: systematic review. BMC Public Health. 2008;8(1):117. doi:10.1186/1471-2458-8-117

14. Denic A, Glassock RJ, Rule AD. Structural and functional changes with the aging kidney. Adv Chronic Kidney Dis. 2016;23(1):19-28. doi:10.1053/j.ackd.2015.08.004

15. Wilson FP, Yang W, Machado CA, et al. Dialysis versus nondialysis in patients with AKI: a propensity-matched cohort study. Clin J Am Soc Nephrol. 2014;9(4):673-681. doi:10.2215/CJN.07630713

16. Sutherland SM, Ji J, Sheikhi FH, et al. AKI in hospitalized children: epidemiology and clinical associations in a national cohort. Clin J Am Soc Nephrol. 2013;8(10):1661-1669. doi:10.2215/CJN.00270113

17. Kaaviya R, Vadivelan M, Balamurugan N, Parameswaran S, Thabah M. Community acquired AKI: a prospective observational study from a tertiary level hospital in Southern India. Indian J Nephrol. 2019;29(4):254-260. doi:10.4103/ijn.IJN_238_18 
18. Tollitt J, Bennett N, Darby D, et al. The importance of acute kidney injury in suspected community acquired infection. PLoS One. 2019;14(5):e0216412. doi:10.1371/journal.pone.0216412

19. Gonzalez LS 3rd, Spencer JP. Aminoglycosides: a practical review. Am Fam Physician. 1998;58(8):1811-1820.

20. Becker B, Cooper MA. Aminoglycoside antibiotics in the 21st century. ACS Chem Biol. 2013;8(1):105-115. doi:10.1021/cb3005116

21. Gyamlani G, Potukuchi PK, Thomas F, et al. Vancomycin-associated acute kidney injury in a large veteran population. Am J Nephrol. 2019;49(2):133-142. doi:10.1159/000496484
22. Arora P, Pourafkari L, Visnjevac O, Anand EJ, Porhomayon J, Nader ND. Preoperative serum potassium predicts the clinical outcome after non-cardiac surgery. Clin Chem Lab Med. 2017;55 (1):145-153. doi: $10.1515 / \mathrm{cclm}-2016-0038$

23. Kovesdy CP, Regidor DL, Mehrotra R, et al. Serum and dialysate potassium concentrations and survival in hemodialysis patients. Clin J Am Soc Nephrol. 2007;2(5):999-1007. doi:10.2215/CJN.04451206

24. Wilson FP, Yang W, Feldman HI. Predictors of death and dialysis in severe AKI: the UPHS-AKI cohort. Clin J Am Soc Nephrol. 2013;8 (4):527-537. doi:10.2215/CJN.06450612

\section{Publish your work in this journal}

The International Journal of General Medicine is an international, peer-reviewed open-access journal that focuses on general and internal medicine, pathogenesis, epidemiology, diagnosis, monitoring and treatment protocols. The journal is characterized by the rapid reporting of reviews, original research and clinical studies across all disease areas. The manuscript management system is completely online and includes a very quick and fair peer-review system, which is all easy to use. Visit http://www.dovepress.com/ testimonials.php to read real quotes from published authors.

Submit your manuscript here: https://www.dovepress.com/international-journal-of-general-medicine-journal 